\title{
Neurons in the Rat Lateral Hypothalamic Area Integrate Information from the Gastric Vagal Nerves and the Cerebellar Interpositus Nucleus
}

\author{
Yue-Ping Zhang ${ }^{a, c}$ Jing-Ning Zhu ${ }^{a}$ Kun Chen ${ }^{a}$ Hong-Zhao Li ${ }^{a}$ \\ Jian-Jun Wang ${ }^{a, b}$ \\ a Department of Biological Science and Technology and State Key Laboratory of Pharmaceutical Biotechnology, \\ School of Life Sciences, Nanjing University; ${ }^{b}$ Key Laboratory of Resource Biotechnology of Jiangsu Province, \\ School of Life Sciences, Nanjing Normal University, Nanjing, and ${ }^{\mathrm{C}}$ Pediatric Department of Xijing Hospital, \\ Fourth Military Medical University, Xi'an, PR China
}

\section{Key Words}

Gastric vagal nerves · Cerebellar interpositus nucleus • Lateral hypothalamic area · Glycemia-sensitive neuron • Cerebellohypothalamic projections - Feeding control

\begin{abstract}
Previous investigations have demonstrated that the neuronal activity in the lateral hypothalamic area (LHA) is respectively modulated by afferent inputs from the gastric vagal nerves innervating the upper gastrointestinal tract, as well as the cerebellar interpositus nucleus (IN). The aim of this study was to examine whether the gastric vagal and cerebellar IN inputs converge onto single LHA neurons in rats, especially those sensitive to glycemia. Of the 114 LHA neurons recorded, 60 (52.6\%) and 51 $(44.7 \%)$ responded to gastric vagal and cerebellar IN stimulation, respectively. Of the 60 LHA neurons responsive to gastric vagal stimulation, 30 also responded to the cerebellar IN stimulus, indicating a convergence of gastric vagal and cerebellar inputs onto single hypothalamic cells. When the gastric vagal nerves and cerebellar IN were stimulated simultaneously, a summation of the responses was observed in all 6 neurons tested. Moreover, of 24 neurons that responded to both the gastric vagal and cerebellar IN stimuli, 15 (62.5\%) were identified as glycemia-sensitive. These results demonstrate
\end{abstract}

that the visceral information transmitted by the gastric vagal nerves and the somatic information forwarded by the cerebellar IN converge onto single LHA neurons, especially those sensitive to glycemia. The findings also suggest that integration of somatic-visceral responses related to short-term feeding regulation may take place in the LHA.

Copyright $(2005$ S. Karger AG, Basel

\section{Introduction}

The hypothalamus is a central area where many peripheral signals and neural pathways that relate to autonomic functions and motivated behaviors converge [1, 2]. The lateral hypothalamic area (LHA) is closely associated with the initiation and regulation of feeding [3-8], and it is well known that neurons in the LHA can sense the blood glucose concentration [9, 10]. A fall in blood glucose may induce hunger and initiate feeding $[9,11]$, and a growing body of evidence suggests that those neurons sensitive to glycemia integrate food and water intake, various forms of social behavior and neuroendocrine signals involved in the regulation of homeostasis of blood glucose, feeding and other physiological functions $[12,13]$.

\section{KARGER}

Fax +4161306 1234 E-Mail karger@karger.ch www.karger.com
(C) $2005 \mathrm{~S}$. Karger AG, Basel

$1424-862 X / 05 / 0145-0234 \$ 22.00 / 0$

Accessible online at:

www.karger.com/nsg
Jian-Jun Wang

Department of Biological Science and Technology, Mailbox 426, Nanjing University 22 Hankou Road, Nanjing 210093 (PR China)

Tel. +86 2583592714 , Fax +86258359 2714/+862583592705

E-Mail jjwang@nju.edu.cn 
Previous electrophysiological studies in the cat have demonstrated that gastric vagal afferent inputs reach the LHA $[14,15]$, suggesting that meal-related visceral signals induced by food intake and digestion can be transmitted to the LHA via the gastric vagal nerves [16]. However, these studies have not indicated whether the neurons receiving the gastrointestinal signals relayed by the gastric vagal nerves are glycemia-sensitive or not. More recently, the role of the cerebellum in regulating non-somatic visceral processes, feeding behavior included, has received increasing attention [17-25]. Stimulation of the rat cerebellar fastigial nucleus is known to evoke post-synaptic responses and to modulate the discharge of LHA neurons, including the glucose-sensitive/glycemia-sensitive neurons $[20,24]$. Other studies have revealed that glucosesensitive and glucose-insensitive neurons in the LHA respond to stimulation of the cerebellar interpositus nucleus (IN) in cats $[21,22]$. These results suggest that the cerebellum may forward some movement-related information to the LHA through the cerebellohypothalamic projections [26], and may be involved in the regulation of additional non-somatic functions such as food intake.

The above-mentioned findings also suggest that LHA neurons may play a role in the integration of visceral and somatic information conveyed via gastric vagal and cerebellofugal pathways, respectively. However, it is unclear if inputs from the gastric vagal nerves and cerebellar IN converge onto single LHA neurons. One of the purposes of this study is to explore this issue and to examine whether the inputs from these two sources interact with each other. Since feeding behavior is closely related to fluctuation in the blood glucose level $[9,11,27,28]$, the sensitivity of the recorded neurons to intravenous injection of glucose was also investigated. Our results demonstrate a convergence of gastric vagal and cerebellar IN afferent inputs to single LHA neurons, including cells sensitive to glycemia.

\section{Materials and Methods}

\section{Animals and Surgery}

Experiments were performed on 34 adult Sprague-Dawley rats of either sex weighing 250-300 g. The rats were anesthetized with urethane $(0.8 \mathrm{~g} / \mathrm{kg})$ plus $\alpha$-chloralose $(65 \mathrm{mg} / \mathrm{kg})$ given intraperitoneally. A supplemental dose of anesthetic was given usually every $5-6 \mathrm{~h}$. A jugular catheter was placed for intravenous administration of supplemental anesthetic, glucose, normal saline and mannitol. Rectal temperature was maintained at $37 \pm 0.5^{\circ} \mathrm{C}$ by keeping the animals on a heating pad. The ECG was continuously monitored on an oscilloscope. A left femoral catheter filled with normal saline containing heparin $(500 \mathrm{IU} / \mathrm{ml})$ was connected to a self-made blood pressure amplifier through a transducer (BLPR, WPI, USA). Arte- rial pressure was measured throughout the experimental sessions, and no obvious fluctuation was found during and after stimulation of the gastric vagal nerves or cerebellar IN [23-25].

The abdomen was opened by a midline incision and the gastric branches of the dorsal, and ventral vagal trunks were carefully dissected from the surrounding tissues to expose the nerve bundles and were then placed over a bipolar hook electrode. After closing the abdominal incision, the animal was mounted in a stereotaxic frame (1404, David Kopf Instruments, USA). The cisterna magna was opened so that there was no accumulation of cerebrospinal fluid. The scalp was incised and two small circular holes were made in the skull above the LHA and cerebellar IN. After the dura mater was removed, the exposed brain surfaces were covered with $2 \%$ Ringer agar. A concentric bipolar stainless steel electrode (ID $0.1 \mathrm{~mm}$, OD $0.4 \mathrm{~mm}$, tip exposure $0.2 \mathrm{~mm}$ ) was used to stimulate the cerebellar IN. The electrode was stereotaxically placed in the nucleus at $\mathrm{A}-11.6, \mathrm{~L} 1.5-2.5$ and $\mathrm{H} 4.5-5.0$ according to the atlas of Paxinos and Waston [29].

\section{Stimulation, Recording, Data Acquisition and Analysis}

Double-negative rectangular pulses were used to stimulate the gastric vagal nerves (intensity $300-500 \mu \mathrm{A}$, duration $0.5 \mathrm{~ms}$, interval $10 \mathrm{~ms}$ ) $[14,15]$ and cerebellar IN (intensity 50-200 $\mu$ A, duration $0.4 \mathrm{~ms}$, interval $10 \mathrm{~ms}$ ) [24, 25]. The paired pulses were applied to the gastric vagal nerves or/and cerebellar IN every $15 \mathrm{~s}$ up to 100 trials for constructing a peri-stimulus time histogram (PSTH; see below for details). Single neuronal discharges were recorded extracellularly from the LHA (A -2.3 to -2.8, L 1.5-2.0, H 7.5-9.0) [29], which is contralateral to the stimulated cerebellar IN, using a glass electrode filled with $1 \%$ solution of pontamine sky blue in $0.5 \mathrm{M}$ sodium acetate (DC resistance 5-10 M $\Omega$ ). The LHA neuronal discharges were conventionally amplified and monitored on an oscilloscope, and simultaneously fed into a window discriminator. The standard rectangular pulses $(5 \mathrm{~V}, 1.0 \mathrm{~ms})$ triggered from the spikes were sent through an A/D interface (1404 Plus, CED, UK) to a laboratory computer which was used to analyze the data online by the software Spike 2 (CED, UK). If the recorded neuron responded to the gastric vagal stimulation and/or cerebellar IN stimulation, the sensitivity of the cell to intravenous glucose administration $(0.4 M, 0.5 \mathrm{ml} / \mathrm{kg})$, which modified blood glucose in small amounts to simulate normal physiological glycemic fluctuations associated with feeding $[9,10,28]$, was tested further. Cells that showed a specific and significant inhibition in response to the injection of glucose but not to the administration of normal saline $(0.9 \% \mathrm{NaCl}, 0.5 \mathrm{ml} /$ $\mathrm{kg}$, used as an osmotic and non-glycemic control) and mannitol $(0.4 \mathrm{M}, 0.5 \mathrm{ml} / \mathrm{kg}$, used as a volumetric control) were considered to be glycemia-sensitive neurons [10, 21, 22, 24, 25, 28]. In order to examine the possible convergence of gastric vagal and cerebellar IN afferent inputs on the glycemia-sensitive neurons of LHA, in some cells that responded to both the gastric vagal nerves and cerebellar IN stimulation and showed an inhibitory response to glucose administration, the effect of simultaneous stimulation of gastric vagal nerves and cerebellar IN was observed further.

PSTHs of the neuronal discharges were generated by the computer to assess the effects of gastric vagal and cerebellar IN stimulations (sampling interval $=2 \mathrm{~ms}$, sampling length $=1,600 \mathrm{~ms}$, accumulated in 100 trials) and the effects of glucose, saline and mannitol injections (sampling interval $=1 \mathrm{~s}$, sampling length $=900 \mathrm{~s}$, single trial). The response patterns of LHA neurons to the gastric vagal, cerebellar IN and glucose stimulations were determined by 


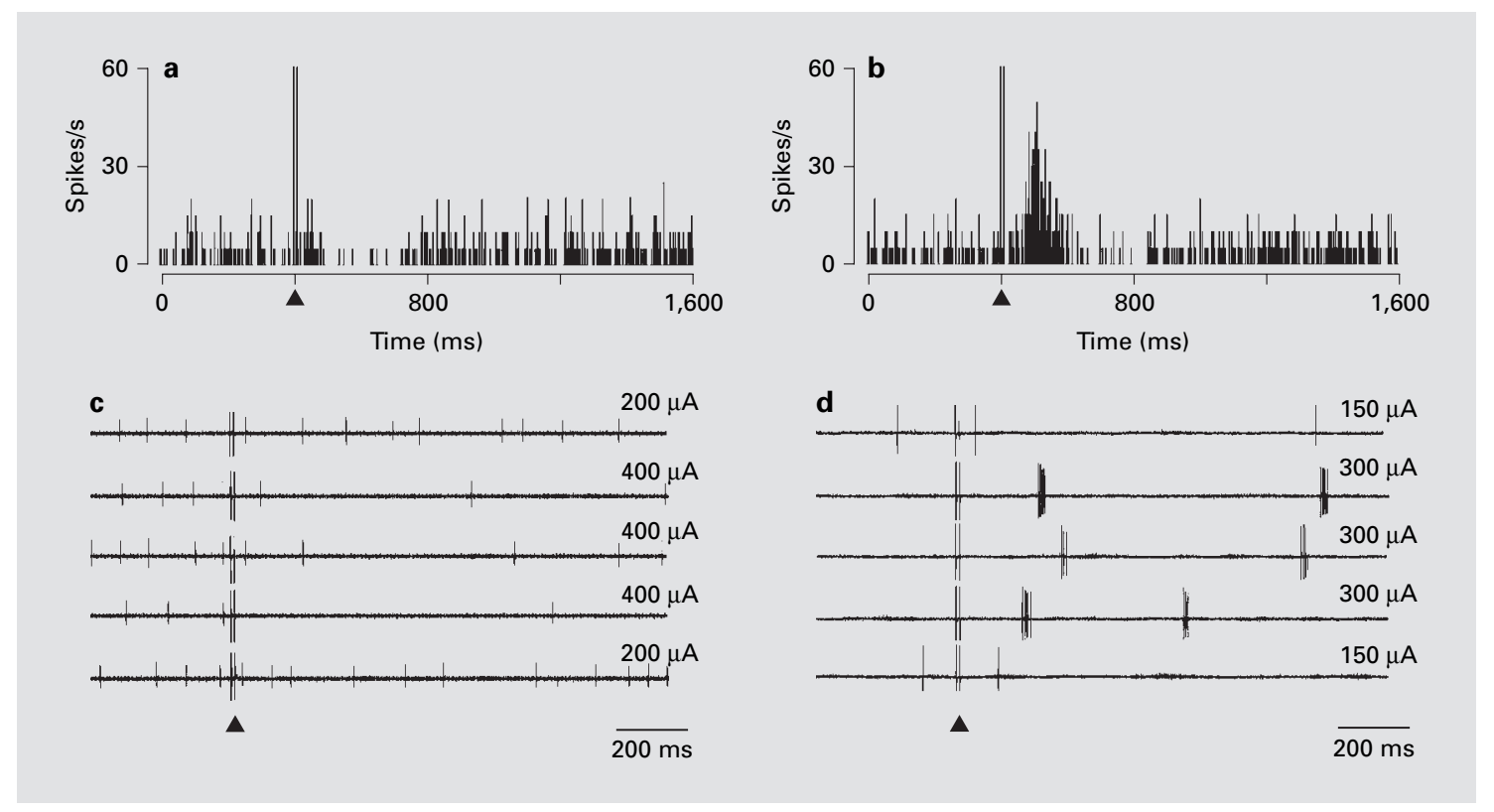

Fig. 1. Two types of responses (phasic and change in firing pattern) of LHA neurons to the stimulation of gastric vagal nerves. a, b PSTHs show the phasic inhibitory and excitatory responses of two LHA cells to the gastric vagal stimulation $(\mathrm{p}<0.05)$, respectively. The arrows under the PSTHs indicate the stimulation artifact. $\mathbf{c}, \mathbf{d}$ The responses of two other LHA neurons to gastric vagal stimulation with a change in discharge pattern are shown. The oscilloscopic traces of the panels were taken respectively from five consecutive raw trails. c The cell responded to the stimulation with a long-lasting decrease in its firing rate. $\mathbf{d}$ The cell changed its firing pattern from single spiking to bursting, consisted of 4 or 5 spikes to each stimulation. Note that the firing pattern of these two cells had no change when the stimulation intensity was sub-threshold (the first and the last oscilloscopic traces of panels $\mathbf{c}$ and $\mathbf{d}$, respectively). Arrows under the last oscilloscopic traces of the panels indicate the stimulation artifact, and the stimulation intensity used for each trial is given at the top right corner of each corresponding trace.

comparing the discharge rate of cells recorded in the post-stimulation response window with the basal firing rate of the cell in the pre-stimulation control window. According to the previously established criteria [20,21, 23-25, 30], inhibition due to stimulation was considered to be significant if the discharge rate in the response window decreased at least to $30 \%$ of the basal rate in the control window, while excitation was considered to be significant if the discharge rate increased to $200 \%$ of the basal rate. Then, the difference between the firing rate in the response window and the firing rate in the control window was statistically evaluated by using Student's t test, and the difference was considered to be significant if the $\mathrm{p}$ values were $<0.05$. For all the cells recorded, the raw data were simultaneously fed into the computer through the A/D interface and collected as records in the form of oscilloscopic traces with a sampling rate of $10 \mathrm{KHz}$.

\section{Histology}

At the end of each experiment, pontamine sky blue was injected into the recording site $(20 \mu \mathrm{A}, 10 \mathrm{~min})$ and DC current $(10 \mu \mathrm{A}, 20 \mathrm{~s})$ was passed through the stimulation electrode to deposit iron at the site of stimulation. The brain was then removed and fixed with $10 \%$ formaldehyde containing $1 \%$ potassium ferrocyanide. A week later, frozen coronal sections ( $80 \mu \mathrm{m}$ thickness) of the brain were prepared to identify the recording and stimulation sites. If the blue dots that indicated the recording and/or stimulating sites were outside the target nuclei, the data were excluded from further analyses.

\section{Results}

\section{LHA Neuronal Responses to Gastric Vagal Stimulation}

A total of 114 LHA neurons were recorded in this study, 60 of which $(52.6 \%)$ responded to the stimulation of gastric vagal nerves (table 1). Of the 60 responsive neurons, 53 responded phasically $(53 / 60,88.3 \%)$ to the stimulation. This type of response includes a transient decrease or increase in discharge rate within a relatively constant latency (40-126 ms) following the gastric vagal stimulation (fig. 1a, b). The remaining 7 responsive neurons $(7 / 60,11.7 \%)$ showed a change in their firing pattern to the stimulation (fig. 1c, d). 
Table 1. The responses of 114 LHA neurons to gastric vagal and cerebellar IN stimulation

\begin{tabular}{lll}
\hline & $\begin{array}{l}\text { Neurons responding to } \\
\text { gastric vagal stimulation }\end{array}$ & $\begin{array}{l}\text { Neurons responding to } \\
\text { cerebellar IN stimulation }\end{array}$ \\
\hline Phasic responses & $53(53 / 60,88.3 \%)$ & $51(51 / 114,44.7 \%)$ \\
Inhibition & $39(39 / 53,73.6 \%)$ & $36(36 / 51,70.6 \%)$ \\
Excitation & $14(14 / 53,26.4 \%)$ & $15(15 / 51,29.4 \%)$ \\
Tonic responses & $7(7 / 60,11.7 \%)$ & - \\
No responses & 54 & 63 \\
\hline
\end{tabular}

${ }^{1}$ The total number of LHA neurons responding to gastric vagal stimulation, including phasic and tonic responsive cells, was 60 $(60 / 114,52.6 \%)$.

Among the 53 neurons that responded to the gastric vagal stimulation with a phasic response, $39(73.6 \%)$ were inhibited (including inhibitory-excitatory responses, $\mathrm{p}<$ 0.05; fig. 1a), and 14 (26.4\%) were excited (including excitatory-inhibitory responses, $\mathrm{p}<0.05$; fig. $1 \mathrm{~b}$ ). The mean latencies of the inhibitory and excitatory responses were $89.7 \pm 20.1$ and $97.9 \pm 21.8 \mathrm{~ms}$ (mean $\pm \mathrm{SD}$ ), respectively. There was no significant difference in the mean latencies of the inhibitory and excitatory responses $(p>$ 0.05). On 5 phasically responsive LHA neurons, the relationship between the stimulation intensity and the response magnitude was further examined. The results showed that these neurons exhibited stimulation intensity-dependent responses to the gastric vagal stimulation, revealing the existence of recruitment of the gastric vagal afferent inputs to the LHA neurons (fig. 2a-c).

In the remaining 7 LHA neurons whose firing patterns were modified in response to stimulation of gastric vagal nerves, 5 responded with a long-lasting decrease $(n=3)$ or increase $(n=2)$ in their firing rate (fig. 1c) and 2 changed their firing pattern from single and discrete spiking to burst discharging consisting of 2-5 spikes (fig. 1d). Once the stimulus was stopped or the intensity of stimulation was reduced to subthreshold, the changed firing patterns of these neurons reverted to their original discharge manner (fig. 1c, d), suggesting that the responses were stimulus-related. These results indicate that neurons in the LHA may show different response characteristics to the feeding-related visceral signals transmitted by the gastric vagal nerves.

We successfully completed the glycemia-sensitivity tests on 45 of the 60 LHA neurons that responded to the gastric vagal stimulation, although the remaining 15 cells were lost during the test session. On the basis of the test-

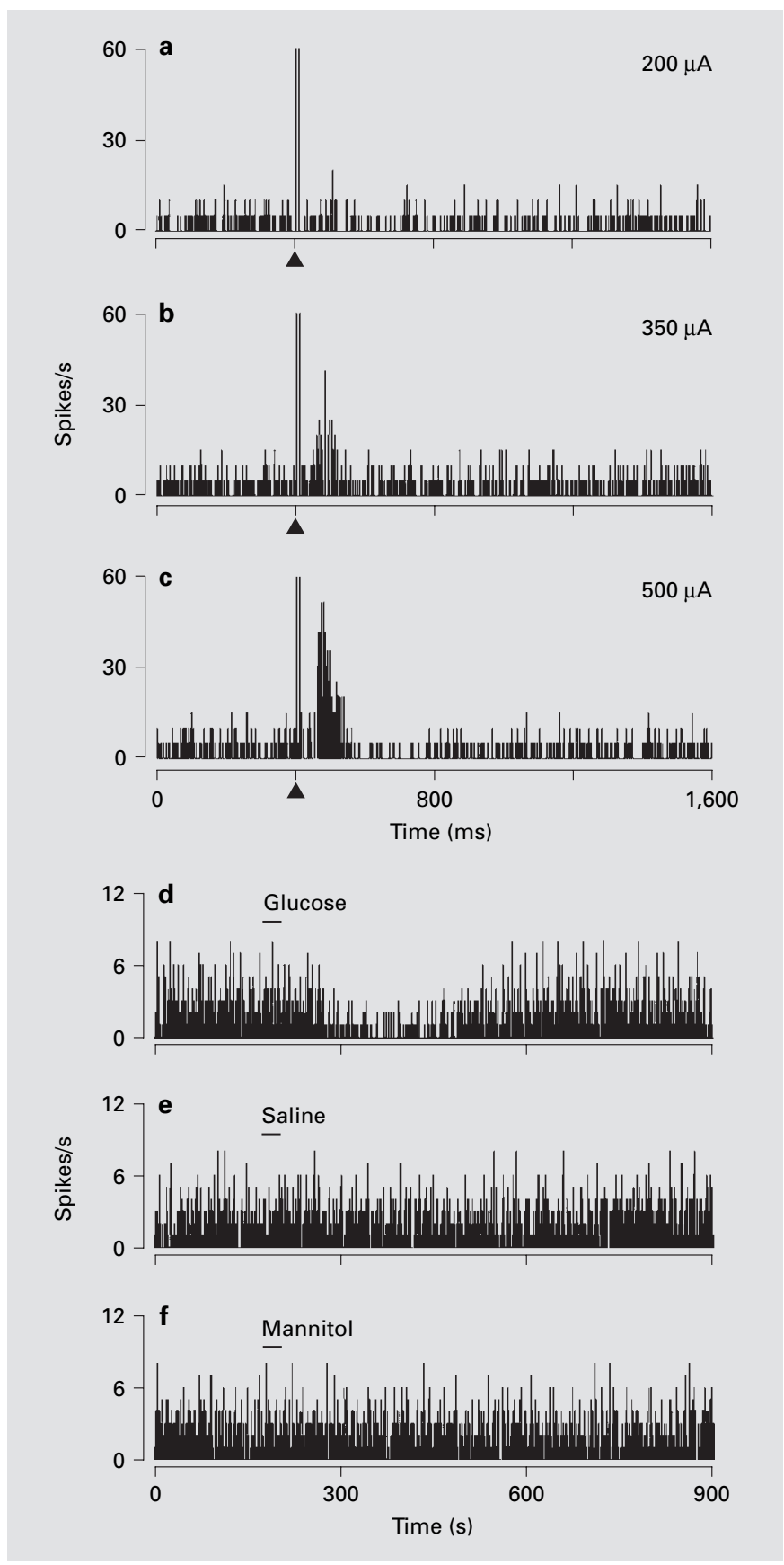

Fig. 2. An LHA neuron that responded to the gastric vagal stimulation showing inhibition to the intravenous glucose administration. a-c PSTHs show the intensity-dependent excitatory responses of the LHA neuron to gastric vagal stimulations at different intensities $(\mathrm{p}<0.05)$. The stimulation intensity used for each experiment is given at the top right corner of each corresponding PSTH. The cell exhibited a specific inhibitory response to glucose administration $(\mathrm{p}<0.05 ; \mathbf{d})$, but had no response to the saline and mannitol injection $(p>0.05 ; \mathbf{e}, \mathbf{f})$, suggesting that the cell was a glycemia-sensitive neuron. Arrows under PSTHs (a-c) indicate the stimulation artifact, and the bars in PSTHs (d-f) show the glucose, saline and mannitol injection, which lasted for $30 \mathrm{~s}$. 

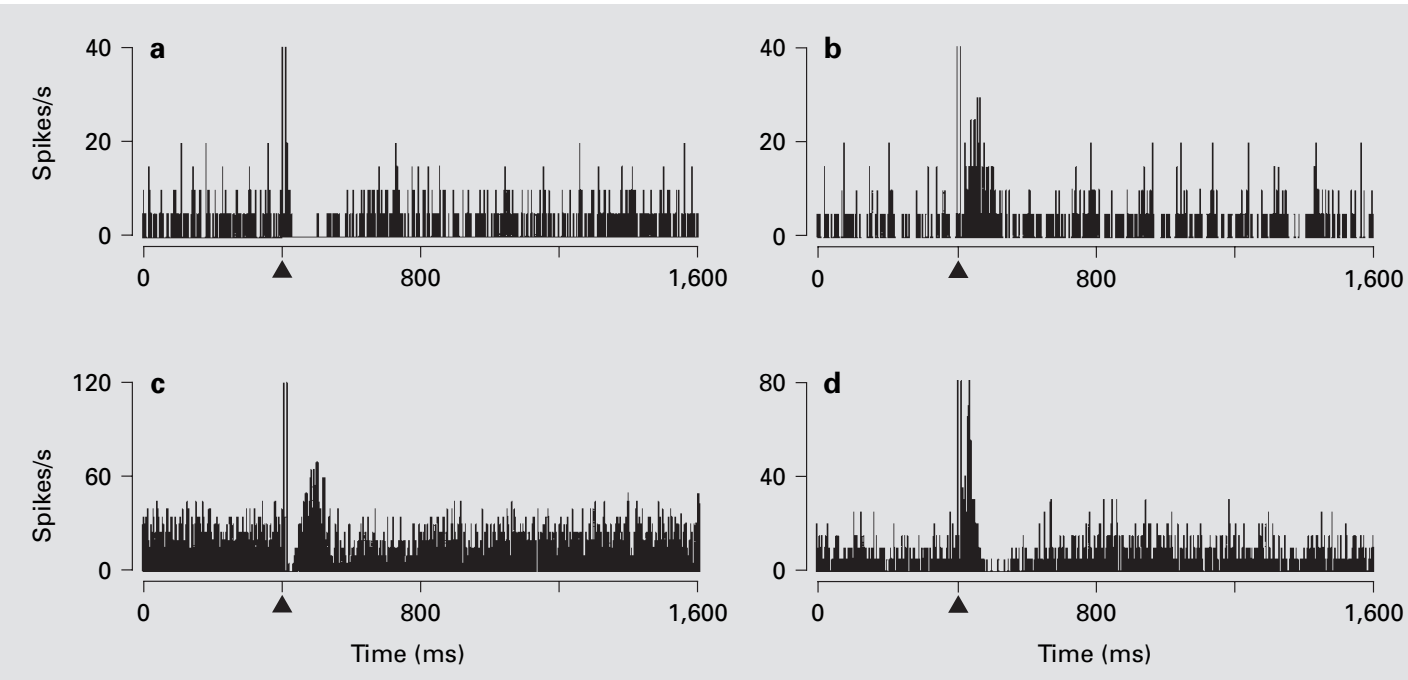

Fig. 3. Four types of responses of LHA neurons to the cerebellar IN stimulation. PSTHs show inhibitory (a), excitatory (b), inhibitory-excitatory (c) and excitatory-inhibitory (d) responses of four different LHA neurons following IN stimulation $(\mathrm{p}<0.05)$. Arrows under the PSTHs indicate the stimulation artifact.

ed neurons showing a specific and significant inhibitory response $(\mathrm{p}<0.01)$ to the intravenous injection of glucose but no response to the administration of normal saline and mannitol, $27(27 / 45,60 \%)$ were found to be glycemiasensitive neurons. One such case is illustrated in figure $2 \mathrm{~d}-\mathrm{f}$. The result demonstrates that glycemia-sensitive neurons in the LHA receive inputs from gastric vagal nerves.

\section{LHA Neuronal Responses to Cerebellar IN Stimulation}

Among the 114 LHA neurons recorded in this study, $51(51 / 114,44.7 \%)$ responded to the contralateral cerebellar IN stimulation (table 1). Of the 51 LHA neurons, $36(36 / 51,70.6 \%)$ showed inhibitory responses (including inhibitory-excitatory responses) and 15 (15/51, 29.4\%) exhibited excitatory responses (including excitatory-inhibitory responses; fig. 3). Moreover, 33 of the 36 inhibited neurons $(91.7 \%)$ showed a short latency (range 2$14 \mathrm{~ms}$ ) response to the IN stimulation with a mean latency of $9.1 \pm 2.8 \mathrm{~ms}$, and the other 3 inhibited neurons $(8.3 \%)$ responded to the IN stimulation with a long latency of $>20 \mathrm{~ms}$. Fourteen of the 15 excited neurons had a short latency excitatory response of 4-18 $\mathrm{ms}$ and the mean latency was $12.6 \pm 3.2 \mathrm{~ms}$, and another one showed a long latency of $50 \mathrm{~ms}$ to the stimulation. The characteristics of the responses of these responsive LHA neurons to the cerebellar IN stimulation were consistent with pre- vious studies in cats [21, 22]. Furthermore, the results from 5 tested LHA neurons showed that the cells responded to the cerebellar IN stimulation in a stimulus intensitydependent manner (fig. $4 \mathrm{a}-\mathrm{c}$ ).

We also conducted glycemia-sensitivity tests on 36 of the 51 LHA neurons that responded to the cerebellar IN stimulation. The results showed that 21 of the examined cells $(21 / 36,58.3 \%)$ were sensitive to glycemia (fig. 4d-f).

\section{LHA Neuronal Responses to Simultaneous Stimulation of the Gastric Vagal Nerves and Cerebellar IN}

Interestingly, in this study 30 of the 60 LHA neurons responsive to gastric vagal stimulation also responded to cerebellar IN stimulation. Twenty-one (70\%) of these 30 neurons responded in the same direction to gastric vagal and cerebellar IN stimulation (i.e., the neurons showed either inhibitions or excitations to both of the stimulations); the remaining 9 cells responded in a different direction to the gastric vagal and cerebellar IN stimulation (9/30, $30 \%$; i.e., inhibition/excitation or excitation/inhibition). These results suggest the possibility that convergence of gastric vagal and cerebellar IN inputs may happen in the same LHA neuron. To examine the possibility, the effect of simultaneous stimulation of gastric vagal nerves and cerebellar IN was observed in 6 cells. The results demonstrated that simultaneously stimulating these 


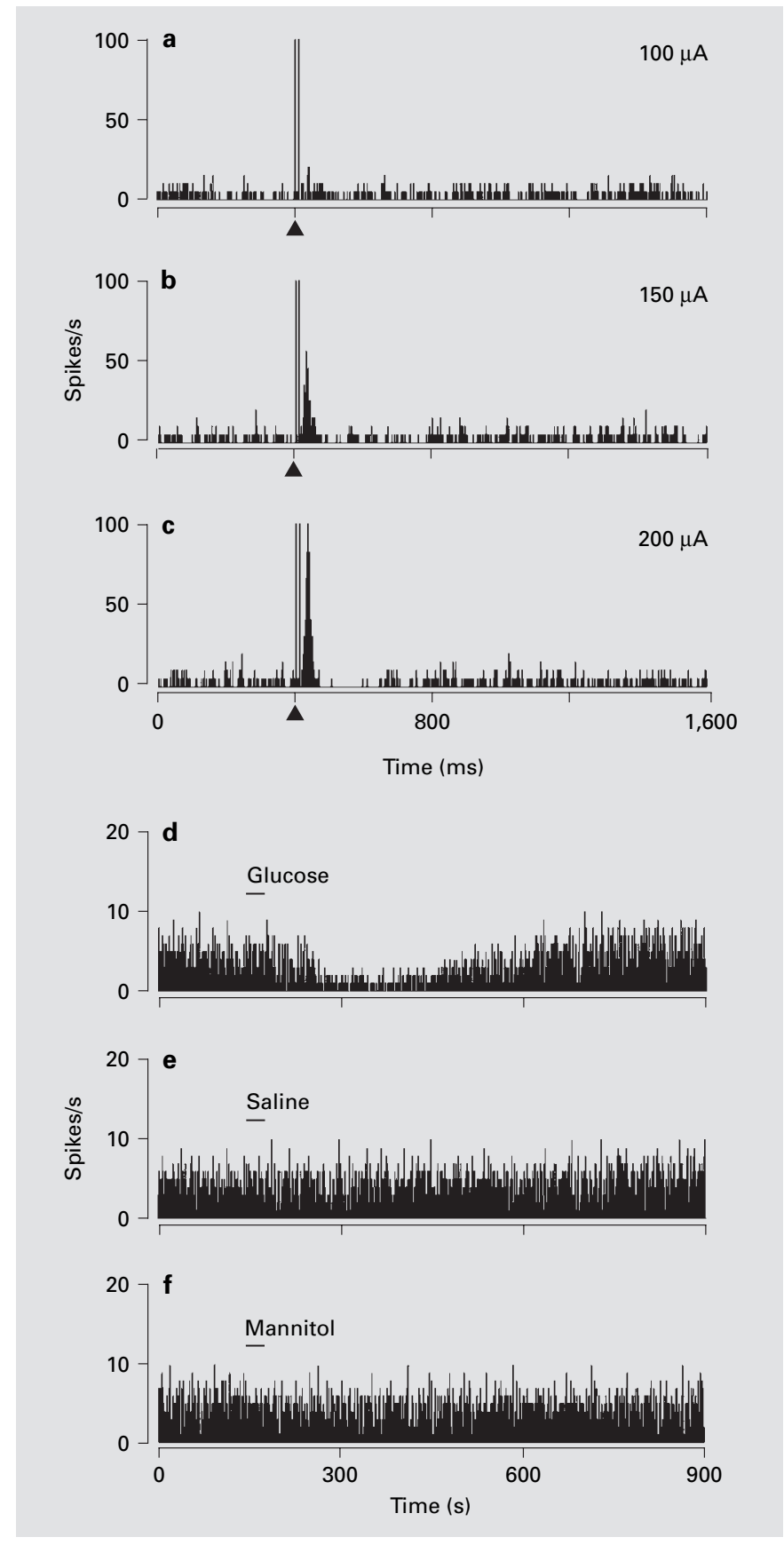

Fig. 4. An LHA neuron that responded to the cerebellar IN stimulation showing inhibition to the intravenous glucose administration. The cell exhibited an intensity-dependent excitatory responses to the IN stimulations at different intensities $(p<0.05$; $\mathbf{a}-\mathbf{c})$. In addition, in company with the increase in stimulation intensity $(200 \mu \mathrm{A})$, the cell also showed inhibition following the excitatory response $(\mathrm{p}<0.05 ; \mathbf{c})$. The stimulation intensity is given at the top right corner of each corresponding PSTH. The glycemia-sensitivity test showed that the neuron responded to the glucose injection with a specific inhibition $(\mathrm{p}<0.05$; d $)$, but not to the saline and mannitol application $(\mathrm{p}>0.05 ; \mathbf{e}, \mathbf{f})$, suggesting that the cell was glycemia-sensitive. The layout of this figure is the same as figure 2 .

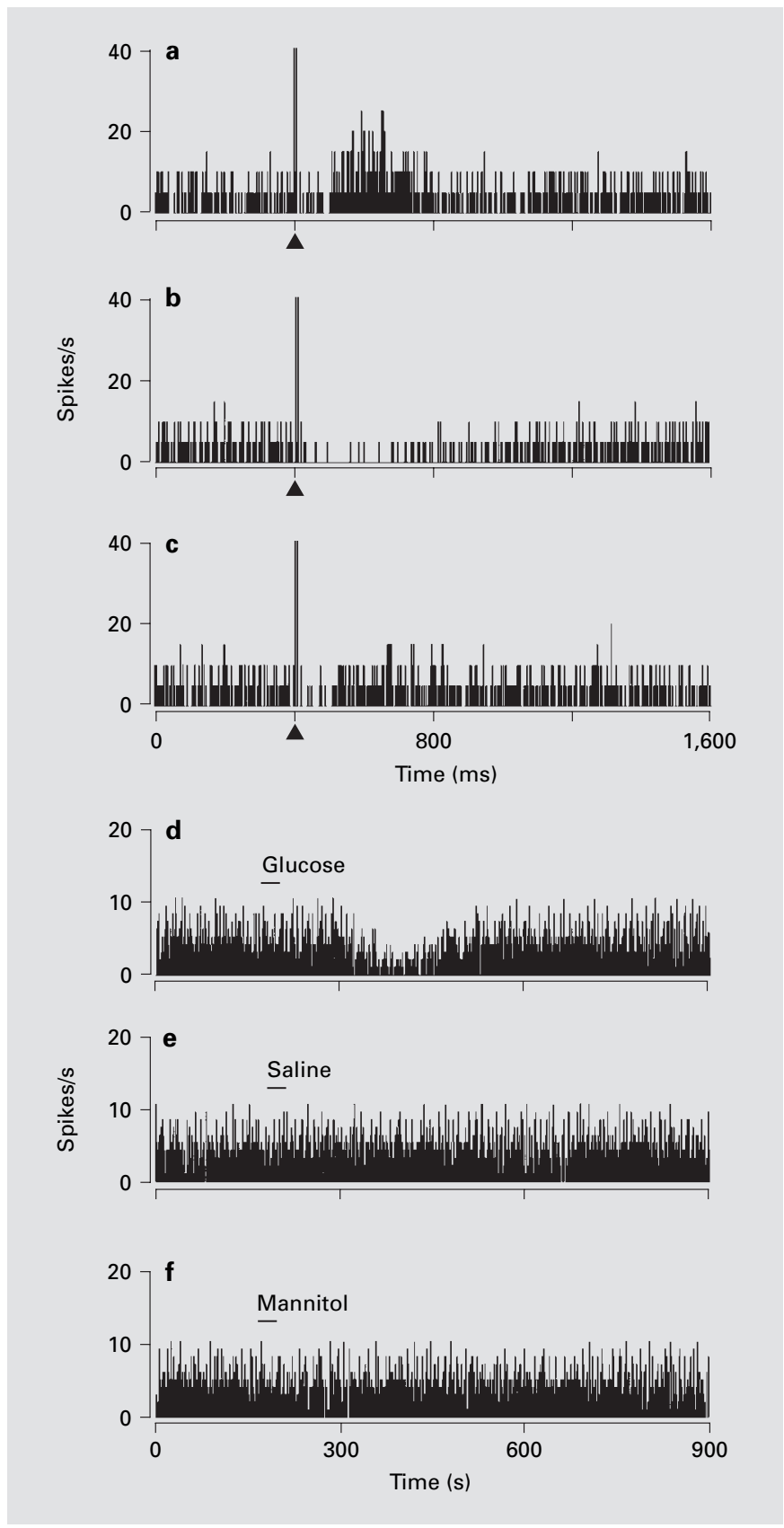

Fig. 5. An LHA neuron that received convergent inputs from gastric vagal nerves and cerebellar IN showing inhibition following an intravenous glucose administration. a Stimulation of the gastric vagal nerves resulted in the recorded LHA neuron an excitation $(p<0.05)$. b Stimulation of the cerebellar IN induced an inhibitory response in the neuron $(\mathrm{p}<0.05)$. c Simultaneous stimulation of the gastric vagal nerves and cerebellar IN did not elicit a significant cell response $(p>0.05)$, suggesting the existence of summation of the gastric vagal- and cerebellar IN-induced responses on the LHA cell. The test of intravenously injecting glucose (d), saline (e) and mannitol (f) revealed that the cell was a glycemia-sensitive neuron. a-c Arrows indicate the stimulation artifact. d-f Bars show the glucose, saline and mannitol injections, which lasted for $30 \mathrm{~s}$. 


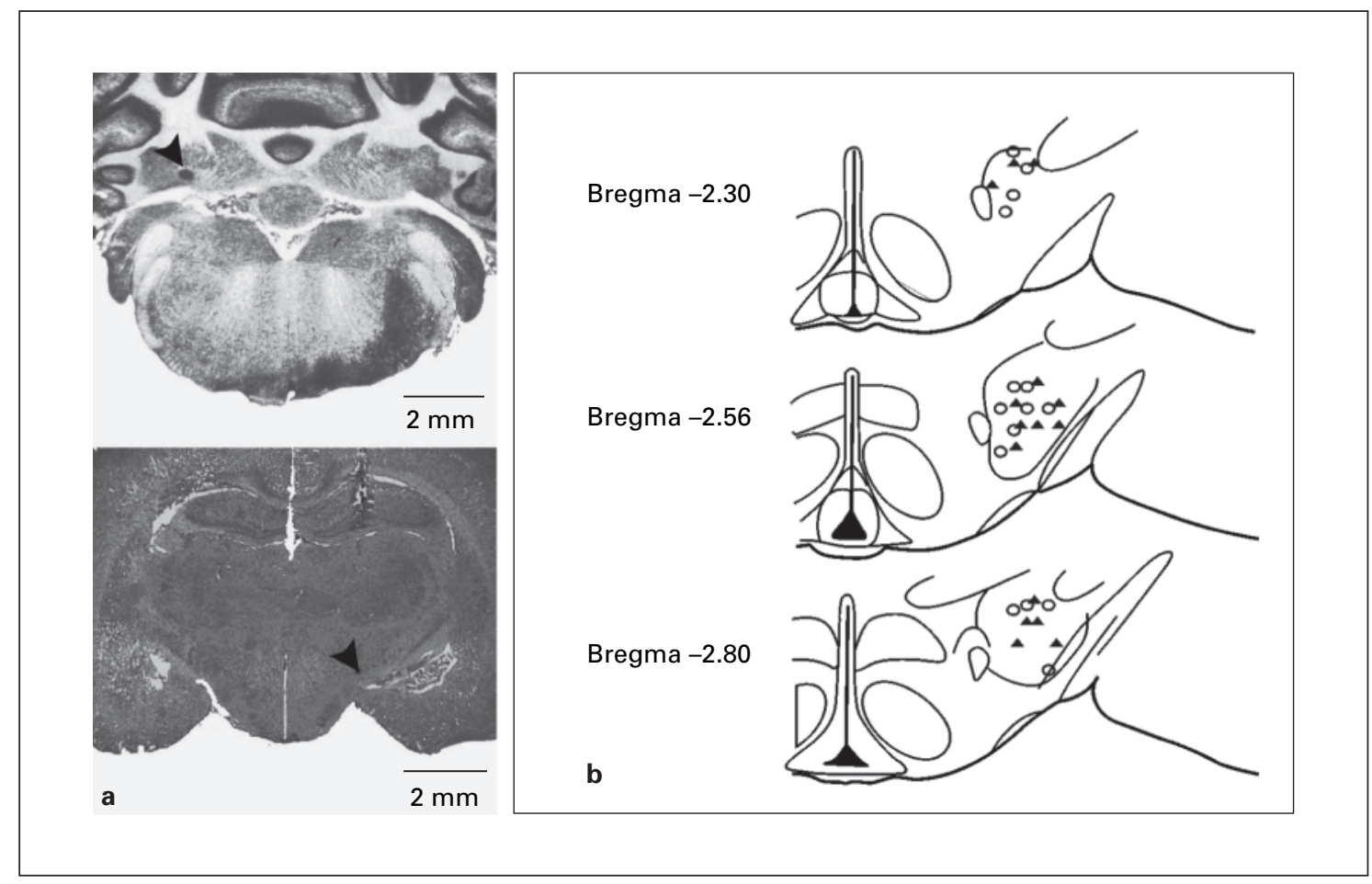

Fig. 6. The distribution of neurons that responded to both gastric vagal and cerebellar IN stimulation in the LHA. a A transverse section of the cerebellum ( $80 \mu \mathrm{m}$ in thickness) showing the stimulation site in the IN (indicated by the arrowhead) and a transverse section of the hypothalamus ( $80 \mu \mathrm{m}$ in thickness) presenting the recording site (indicated by the arrowhead). b Histological reconstruction showing the sites of neurons that responded to gastric vagal and cerebellar IN stimulation in the LHA. Three transverse sections of the hypothalamus corresponding to the planes Bregma $-2.30 \mathrm{~mm},-2.56 \mathrm{~mm}$ and $-2.80 \mathrm{~mm}$ of the atlas of Paxinos and Waston [29] are presented, respectively. The recorded cells were either glycemia-sensitive $(\boldsymbol{\Delta})$ or glycemia-insensitive $(\bigcirc)$ neurons.

Table 2. Glycemia sensitivity of the LHA neurons responding to gastric vagal or/and cerebellar IN stimulation

\begin{tabular}{llll}
\hline Type of neurons & $\begin{array}{l}\text { Neurons responding } \\
\text { to gastric vagal } \\
\text { stimulation }(\mathrm{n}=45)\end{array}$ & $\begin{array}{l}\text { Neurons responding } \\
\text { to cerebellar IN } \\
\text { stimulation }(\mathrm{n}=36)\end{array}$ & $\begin{array}{l}\text { Neurons responding } \\
\text { to stimulations of } \\
\text { gastric vagal nerves and } \\
\text { cerebellar IN (n = 24) }\end{array}$ \\
\hline $\begin{array}{l}\text { Glycemia-sensitive neurons } \\
\text { Glycemia-insensitive neurons }\end{array}$ & $27(60 \%)$ & $21(58.3 \%)$ & $15(62.6 \%)$ \\
$18(40 \%)$ & $15(41.7 \%)$ & $9(37.4 \%)$ \\
\hline
\end{tabular}

two sites caused a summation of responses in all 6 LHA neurons tested, i.e., the responses of these neurons to simultaneous stimulation of the gastric vagal and cerebellar IN were attenuated in comparison with either stimulating gastric vagal or cerebellar IN alone if the responses of the neurons to stimulation of vagal and cerebellar IN were not in the same response direction (fig. 5); while the responses induced by simultaneously stimulating the two sites were more pronounced if the original responses were in the same direction (data not shown).

To further examine whether the gastric vagal and cerebellar IN inputs converge onto the same single LHA glycemia-sensitive neuron, we conducted a glycemia-sensitive test on 24 of 30 LHA neurons that responded to both the gastric vagal and cerebellar IN stimulation. The results showed that 15 of 24 cells $(62.5 \%)$ were glycemia- 
sensitive neurons (fig. 5d-f), demonstrating that the afferent inputs from the gastric vagal nerves and cerebellar IN converge onto a single glycemia-sensitive neuron of the LHA, and that most LHA neurons which responded to gastric vagal and/or cerebellar IN stimulation were sensitive to glycemia (table 2).

\section{Distribution of Responsive Neurons in the LHA}

Histologically verified recording sites for the 30 LHA neurons that responded to both the gastric vagal nerves and cerebellar IN are reconstructed in figure 6. Fifteen of them were glycemia-sensitive neurons. It can be seen from figure 6 that the neurons responsive to both vagal and cerebellar IN stimulation and the glycemia-sensitive neurons were scattered over the LHA and showed no specific regional difference.

\section{Discussion}

It has been demonstrated that the gastric vagal fibers innervating the upper gastrointestinal tract can transmit three classes of meal-related information to the brain: mechanical distention of the lumen or gut contraction, chemical properties of luminal contents and gut peptides. Each of them reduces the meal size [16, 31]. This fact strongly suggests that gastrointestinal vagal afferent fibers may convey the negative-feedback signals to central areas, such as the nucleus tractus solitarius and hypothalamus, which play important roles in the control of food intake. However, little is known about the exact relationship between the peripherally activated vagal signals and the hypothalamic control of feeding behavior. The present study revealed that $52.6 \%$ of the recorded LHA neurons responded to gastric vagal stimulation and that most of the cells were inhibited. These results obtained from experiments on rats are comparable with previous findings from cats $[14,15]$. Although the sub-diaphragmatic vagal trunks contain afferent and efferent components, a histological and functional study has well documented that at least $80 \%$ of the vagal fibers are afferent [32]. Besides, our previous study [25] revealed that vagotomy abolished the similar vagal-induced neuronal responses of the ventromedial hypothalamic nucleus (VMN), indicating that the stimulation may not activate any other spinal afferent pathways to the hypothalamus. In this study, the long response latencies of the LHA neuronal responses to gastric vagal stimulation suggested that inputs from the gastrointestinal tract to the hypothalamus are polysynaptic. Yuan and Barber $[14,15]$ demonstrated

LHA Integration of Gastric Vagal and Cerebellar Inputs that they were relayed by the nucleus tractus solitarius. In addition, data from this study also showed that the responses of LHA neurons to gastric vagal stimulation were diverse, i.e., besides the transient phasic response, some LHA cells responded to gastric vagal stimulation with a change in their firing pattern. The result indicates that LHA neurons may employ various spiking patterns to encode different meal-related information that has arisen from the gastrointestinal tract for accurate transmission and processing of visceral sensory inputs in feeding control. It is thus suggested that the inputs from the gastrointestinal tract to LHA neurons, conveyed by gastric vagal afferent fibers, may be an important component of the negative feedback regulation of feeding.

Feeding behavior is closely linked to fluctuation in the blood glucose level [9-11,27], and it is well documented that about $40 \%$ of the neurons in the LHA are glycemiasensitive neurons [11,33] which may be involved in meal onset triggered by a blood glucose drop [28]. In this study, the correlation between the gastric vagal afferent inputs and the glycemia-sensitive neurons in the LHA was further documented. Among 45 LHA neurons that responded to the gastric vagal stimulation, $27(60 \%)$ were found to be sensitive to glycemia. The results prove that the gastrointestinal inputs conveyed by the gastric vagal afferent fibers mostly reach those glycemia-sensitive neurons of the LHA. Therefore, the modulatory effect of the gastric vagal afferent inputs on the LHA glycemia-sensitive neurons may play an important role in the short-term regulation of feeding behavior.

On the other hand, the cerebellar influence on feeding control has also been revealed successively during the past decade. Behavioral studies have demonstrated that animals with lesions of cerebellar cortex showed an alteration in food intake behavior, a disturbance in nutritional utilization, as well as a decrease in body weight [ 18 , $34,35]$. A recent study using the technique of functional magnetic resonance imaging provided more direct evidence of cerebellar involvement in feeding control, indicating that the cerebellum was indeed activated by glucose intake in human subjects without any accompanying motor behavior [17]. Although the connections between the cerebellar fastigial nucleus and the glucose-sensitive/ glycemia-sensitive neurons in the LHA have been disclosed [20, 22, 24], the cerebellar IN, which developed later in vertebrate phylogeny for adapting to the need for more precise control of distal limb muscles and seems to be more important in reaching and grasping food than fastigial control of axial and facial musculatures [36], has been neglected in research on cerebellohypothalamic pro- 
jections. Thus, it is necessary to investigate the possibility of projections arising from the cerebellar IN to those LHA glycemia-sensitive neurons. In this study, 44.7\% (51/114) LHA neurons responded to the contralateral cerebellar IN stimulation mostly with a short response latency (range 2-14 ms), suggesting that the projections from cerebellar IN to LHA may be direct (monosynaptic). These response characteristics of LHA neurons to cerebellar IN stimulation are comparable with previous results obtained from the cat [21]. Furthermore, our result that $21(58.3 \%)$ LHA cells, which responded to the cerebellar IN stimulation, were found to be glycemia-sensitive neurons indicates that the neuronal activities of glycemia-sensitive neurons in the LHA can be modulated by cerebellar IN inputs. Besides, it was recently observed that the glycemia-sensitive neurons of the VMN, another important central area controlling food intake and satiety, were impinged by the cerebellar IN inputs [25]. All of these data support the notion that the neuronal activities of the cerebellar cortex and deep nuclei (including fastigial and interpositus nuclei) may represent some somatic information related to food seeking and intake, and the information may be further forwarded to the feedingassociated hypothalamic structures, particularly those glycemia-sensitive neurons in the structure $[21,22,24$, 25]. Therefore, it can be proposed that the cerebellum may be actively involved in the short-term regulation of feeding behavior through mediation of the direct cerebellohypothalamic pathways.

Our previous electrophysiological study has demonstrated a convergence of the gastric vagal nerves and cerebellar IN on single VMN neurons [25], thus whether the gastric vagal and cerebellar IN inputs may converge onto single neurons in the LHA is also an intriguing issue that needs to be investigated further. The results show that $26.3 \%(30 / 114)$ of the LHA neurons recorded in this study responded to both gastric vagal stimulation and cerebellar IN stimulation. When gastric vagal nerves and cerebellar IN were simultaneously stimulated, a summation in response was observed from a given LHA neuron $(6 / 30,20.0 \%$; fig. 5$)$, suggesting that the summation in neuronal response is due to the interaction of convergent inputs from cerebellar IN and gastric vagal nerves. Based on the facts that the LHA neurons are influenced by signals from gastric vagal afferent fibers and the cerebellar $\mathrm{IN}$, and that both of the two kinds of signals converge and interact with each other on single LHA neurons, we postulate that neurons in the LHA may play a larger role in the integration of visceral sensory information and mealrelated somatic information.
The cell's sensitivity to the glucose administration (i.v.) on those LHA neurons that responded to both gastric vagal and cerebellar IN stimulation was also examined in the present study. Among 24 tested LHA neurons, the majority $(15 / 24,62.5 \%)$ was identified to be sensitive to glycemia (table 2). Because the ratio of glycemia-sensitive neurons in the LHA is less than $50 \%[11,33]$, the present result provides strong evidence that gastrointestinal inputs and cerebellar IN inputs not only converge onto single LHA neurons but also mostly converge onto those LHA cells sensitive to glycemia. On the other hand, some studies also showed a strong relationship between the LHA glycemia-insensitive neurons and water- or food-seeking behavior [37-39]. Therefore, it can be inferred that the convergent inputs from the gastric vagal nerves and the cerebellar IN may also be involved in the formation of feeding motion by their effects on the glycemia-insensitive neurons in the LHA. On the basis of the above facts and the present data, we suggest that neurons in the LHA, especially those glycemia-sensitive neurons receiving both gastric vagal inputs and cerebellar IN inputs may integrate the meal-initiating signals (such as hypoglycemia), meal-limiting information (conveyed via gastric vagal afferents), and meal-related somatic information (forwarded by cerebellar IN through cerebellohypothalamic projections), and consequently trigger an appropriate behavioral response related to food intake. Combined with our previous findings that the cerebellar IN and gastric vagal afferent inputs reach and converge onto VMN glycemia-sensitive neurons [25] and the wellestablished functional reciprocal interaction between LHA and VMN on feeding control, we propose that this hypothalamic (including LHA and VMN) integration of the somatic-visceral (cerebellar IN, gastric vagal and glycemia) response may play a critical role in the short-term regulation of feeding behavior.

\section{Acknowledgements}

This work was supported by grants 30370462 and the NSFCRGC Joint Research Scheme (30318004) from the National Natural Science Foundation of China, RFDP grant 20010284021 from the State Educational Ministry of China and grant BK2002083 from the Natural Science Foundation of Jiangsu Province of China. The work was also partially supported by a grant-in-aid of ' 985 Project' from Nanjing University. Dr. Y.P. Zhang was supported by the Postdoctoral Training Program of Nanjing University. 


\section{References}

$\checkmark 1$ Kiyomi N, Taketoshi O, Ryoi T, Motoichi I, Yasuhiro T, Michiaki K: Characteristics of rat lateral hypothalamic neuron responses to smell and taste in emotional behavior. Brain Res 1989;491:15-32.

-2 Snowball RK, Semenenko FM, Lumb BM: Visceral inputs to neurons in the anterior hypothalamus including those that project to the periaqueductal gray: a functional anatomical and electrophysiological study. Neuroscience 2000;99:351-361.

$\checkmark 3$ Bray GA: Afferent signals regulating food intake. Proc Nutr Soc 2000;59:373-384.

4 Jeanningros R: Lateral hypothalamic responses to pre-absorptive and post-absorptive signals related to amino acid ingestion. J Auton Nerv Syst 1984;10:261-268.

$\checkmark 5$ Jeanningros R: Modulation of lateral hypothalamic single unit activity by gastric and intestinal distension. J Auton Nerv Syst 1984;11: $1-11$.

6 Morley JE, Levine AS: The central control of appetite. Lancet 1983;i:398-401.

7 Shiraishi T, Oomura Y, Sasaki K, Wayner MJ: Effects of leptin and orexin-A on food intake and feeding related hypothalamic neurons. Physiol Behav 2000;71:251-261.

$\checkmark 8$ Smith GP: The peripheral control of appetite. Lancet 1983;ii:88-90.

$\checkmark 9$ Himmi T, Boyer A, Orsini JC: Changes in lateral hypothalamic neuronal activity accompanying hyper- and hypoglycemias. Physiol Behav 1988;44:347-354.

- 10 Orsini JC, Wiser AK, Himmi T, Boyer A: Sensitivity of lateral hypothalamic neurons to glycemia level: possible involvement of an indirect adrenergic mechanism. Brain Res Bull 1991;26:472-478.

$>11$ Campfield LA, Smith FJ: Blood glucose dynamics and control of meal initiation: a pattern detection and recognition theory. Physiol Rev 2003;83:25-58.

$>12$ Levin BE, Routh VH, Kang L, Sanders NM, Dunn-Meynell AA: Neuronal glucosensing: what do we know after 50 years? Diabetes 2004;53:2521-2528.

13 Steffens AB, Scheurink AJ, Luiten PG, Bohus $\mathrm{B}$ : Hypothalamic food intake regulating areas are involved in the homeostasis of blood glucose and plasma FFA levels. Physiol Behav 1988;44:581-589.

14 Yuan CS, Barber WD: Hypothalamic unitary responses to gastric vagal input from the proximal stomach. Am J Physiol Gastrointest Liver Physiol 1992;262:G74-G80.
15 Yuan CS, Barber WD: Interactions of gastric vagal and peripheral nerves on single neurons of lateral hypothalamus in the cat. Am J Physiol Gastrointest Liver Physiol 1996;271:G858G865.

16 Schwartz GJ: The role of gastrointestinal vagal afferents in the control of food intake: current prospects. Nutrition 2000; 16:866-873.

17 Liu Y, Gao JH, Liu HL, Fox PT: The temporal response of the brain after eating revealed by functional MRI. Nature 2000;405:1058-1061.

18 Mahler JM: An unexpected role of the cerebellum: involvement in nutritional organization. Physiol Behav 1993;54:1063-1067.

19 Scalera G, Benassi C: Modifications of food intake and body weight after cerebellar cortex lesions in the rat. Med Sci Res 1989;17:105106.

20 Min B, Oomura Y, Katafuchi T: Responses of rat lateral hypothalamic neuronal activity to fastigial nucleus stimulation. J Neurophysiol 1989;61:1178-1184.

-21 Pu YM, Wang JJ, Wang T, Yu QX: Cerebellar interpositus nucleus modulates neuronal activity of lateral hypothalamic area. Neuroreport 1995;6:985-988.

22 Wang JJ, Pu YM, Wang T: Influences of cerebellar interpositus nucleus and fastigial nucleus on neuronal activity of lateral hypothalamic area. Sci Chin (Series C) 1997;40:176-183.

23 Wen YQ, Zhu JN, Zhang YP, Wang JJ: Cerebellar interpositus nuclear inputs impinge on paraventricular neurons of the hypothalamus in rats. Neurosci Lett 2004;370:25-29.

24 Zhang YP, Ma C, Wen YQ, Wang JJ: Convergence of gastric vagal and cerebellar fastigial nuclear inputs on glycemia-sensitive neurons of lateral hypothalamic area in the rat. Neurosci Res 2003;45:9-16.

25 Zhu JN, Zhang YP, Song YN, Wang JJ: Cerebellar interpositus nuclear and gastric vagal afferent inputs reach and converge onto glycemia-sensitive neurons of the ventromedial hypothalamic nucleus in rats. Neurosci Res 2004;48:405-417.

26 Haines DE, Dietrichs E, Mihailoff GA, McDonald EF: The cerebellar-hypothalamic axis: basic circuits and clinical observations. Int Rev Neurobiol 1997;41:83-107.

27 Carrasco M, Portillo F, Larsen PJ, Vallo JJ: Insulin and glucose administration stimulates Fos expression in neurones of the paraventricular nucleus that project to autonomic preganglionic structures. J Neuroendocrinol 2001;13: 339-346.
28 Yettefti K, Orsini JC, Perrin J: Characteristics of glycemia-sensitive neurons in the nucleus tractus solitarii: possible involvement in nutritional regulation. Physiol Behav 1997;61:93100 .

29 Paxinos G, Waston C: The Rat Brain in Stereotaxic Coordinates, ed 4. San Diego, Academic Press, 1998.

30 Lou JS, Bloedel JR: The responses of simultaneously recorded Purkinje cells to the perturbations of the step cycle in the walking ferret: a study using a new analytical method: the realtime postsynaptic response (RTPR). Brain Res 1986;365:340-344.

31 Barber WD, Yuan CS: Gastric vagal evoked and greater-splanchnic evoked unitary responses in the hypothalamus. Am J Physiol Gastrointest Liver Physiol 1993;264:G1133G1141.

32 Agostoni E, Chinnock JE, de Daly MB, Murray JG: Functional and histological studies of the vagus nerve and its branches to the heart, lungs and abdominal viscera in the cat. J Physiol (Lond) 1957;135:182-205.

33 Fukuda M, Ono T, Nishino H, Sasaki K: Independent glucose effects on rat hypothalamic neurons: an in vitro study. J Auton Nerv Syst 1984;10:373-381.

34 Hartmann MJ, Bower JM: Tactile responses in the granule cell layer of cerebellar folium crus IIa of freely behaving rats. J Neurosci 2001;21: 3549-3563.

35 Scalera G: Effects of corticocerebellar lesions on taste preferences, body weight gain, food and fluid intake in the rat. J Physiol (Paris) 1991;85:214-222.

36 Martin JH, Cooper SE, Hacking A, Ghez C: Differential effects of deep cerebellar nuclei inactivation on reaching and adaptive control. J Neurophysiol 2000;83:1886-1899.

37 Aou S, Takaki A, Karadi Z, Hori T, Nishino $\mathrm{H}$, Oomura Y: Functional heterogeneity of the monkey lateral hypothalamus in the control of feeding. Brain Res Bull 1991;27:451-455.

-38 Karadi Z, Oomura Y, Nishino H, Scott TR, Lenard L, Aou S: Complex attribute of lateral hypothalamic neurons in the regulation of feeding of alert rhesus monkeys. Brain Res Bull 1990;25:933-939.

39 Parada MA, Hernandez L, Puig de Parada M, Paez X, Hoebel BG: Dopamine in the lateral hypothalamus may be involved in the inhibition of locomotion related to food and water seeding. Brain Res Bull 1990;25:961-968. 